\title{
A sponsor's experience with the Voluntary Harmonization Procedure for clinical trial applications in the European Union
}

\section{Georgios Amexis and Elmar Schmitt}

A recent interview with Hartmut Krafft ('An audience with... Hartmut Krafft. Nature Rev Drug Discov. 9, 426 (2010)) $)^{1}$ discussed the Voluntary Harmonization Procedure (VHP) for the assessment of multinational clinical trials in the European Union (EU). This pilot programme was established to help address the issues that arose from the implementation of the EU Clinical Trials Directive 2001/20/European Commission (EC) in 2004 into national laws and regulations, which led to divergent review processes for clinical trial applications (CTAs) in the $27 \mathrm{EU}$ member states. It is apparent that a harmonized EU-wide approach for the approval of CTAs is especially important in the context of multinational clinical trials in which various National Competent Authorities (NCAs) and ethics committees are involved. However, there are still different country-specific CTA content formats, evaluation criteria, review timelines and language requirements in place.

Here, as the first commercial sponsor to contribute to the pilot VHP, we describe our experience, which shows that the VHP can be successfully utilized as a valuable strategic tool to achieve faster CTA approvals of multinational clinical trials and to speed up global drug development.

\section{Why does the EU need a new CTA assessment process?}

The scientific community has raised concerns about whether the goals of the EU Directive 2001/20/EC have been accomplished; this Directive was established to advance innovation and research, raise the level of competitiveness of clinical research and improve patient protection by streamlining the scientific assessment of CTAs. Each year $\sim 4,000-6,000$ randomized clinical trial protocols are submitted for assessment by either commercial or academic sponsors to the 27 EU NCAs and numerous local or central ethics committees ${ }^{2}$. Divergent decisions by NCAs on the same clinical trials or various content, format or language requirements, in combination with different assessments made by central and local ethics committees, make it more and more complex to conduct innovative clinical research in the $\mathrm{EU}^{3,4}$. Like other sponsors, Merck Serono have also experienced first-hand that scientific evaluation of multinational CTAs in the EU has led to the unwanted situation of divergent CTA assessments.

To address this issue, in 2004 the EU Heads of Medicines Agencies established the Clinical Trials Facilitation Group (CTFG) to coordinate the implementation of the EU Directive 2001/20/EC. The CTFG started the VHP in the first quarter of 2009 as an alternative to the current national CTA submission procedures. In its current state, the VHP is a pilot programme that allows a sponsor to obtain a consolidated assessment for multinational CTAs or substantial amendments ${ }^{5}$.

\section{Our experience with the VHP}

Merck Serono recognized early on that the VHP offered a unique opportunity to accelerate the assessment of multinational CTAs. However, the main concern from our perspective as a sponsor revolved around the following question: will a parallel scientific assessment by multiple EU health authorities result in a different outcome compared to independent national submissions?

During the pre-VHP submission phase, we established a close working relationship with the CTFG chairman and VHP coordinator to seek guidance on operational and procedural issues. The CTFG demonstrated an open-minded attitude and a clear commitment to working with us by providing helpful guidance to push the CTA assessment ahead. Based on initial CTFG guidance, VHP dossiers were forwarded in parallel in the second quarter of 2009 to all of the NCAs and ethics committees involved. The submitted CTAs for Phase II and Phase III clinical trials (6 and 14 EU member states, respectively) and substantial amendments were both approved after 56 and 19 calendar days, respectively. In our experience, this is $\sim 1$ month quicker than with the current national standard CTA approval process. Furthermore, our concerns about conflicting NCA feedback or postVHP requests did not materialize.

It was interesting to note that EU member states that were not part of the initial VHP assessment but were included as 'add-on countries' followed the VHP decision unanimously and did not raise any questions about the CTA packages during their national submissions. An additional benefit of the VHP from an operational perspective was greater flexibility in resource management by substantially reducing certain contract research organization activities, such as document translations. Furthermore, the elimination of additional sponsor-NCA interactions allowed us to initiate faster patient recruitment and activation of clinical study sites.

Detailed final sponsor reports were submitted in the third quarter of 2009 for each conducted VHP to all NCAs that participated in the VHP process. The CTFG was very interested in receiving sponsor feedback on the VHP process, in general and especially to track the outcome of the national post-VHP approvals.

Overall, in our experience the VHP was a content-driven process in which all stakeholders exhibited a material interest to help generate and approve high-quality CTA dossiers, and we highly encourage fellow sponsors to capitalize on the current VHP process. From a sponsor's perspective, the VHP provides important strategic and operational benefits, such as faster multinational CTA approvals and consolidated scientific CTA assessments. The implementation of the VHP can be viewed as an important step forward in drug development, as it provides a more transparent and coherent approach for CTA evaluation than the current EU and national CTA procedures. However, some drawbacks remain. In this context, we are hopeful that ongoing discussions about whether ethics committees could also be involved in the VHP assessment will lead to a positive outcome ${ }^{1,6}$.

Georgios Amexis and Elmar Schmitt are at the Global Regulatory Oncology/Regulatory Oncology EU, Merck KGaA, Frankfurter Strasse 250, 64293 Darmstadt, Germany.

Correspondence to G.A e-mail: georgios.amexis@merck.de Hughes, B. An audience with... Hartmut Krafft. Nature Rev Drug Discov. 9, 426 (2010).

European Commission. Assessment of the functioning of the 'Clinical Trials Directive' 2001/20/EC public consultation paper. European Commission 'online], http://ec.europa.eu/health/files/clinicaltrials/ docs/2009 1009 public-consultation-paper.pdf
(2009). 
CORRESPONDENCE

3. Garattini, S. (European Commission). Can we facilitate multinational investigator-driven trials? European Commission [online], http://ec.europa.eu/research

health/medical-research/pdf/idct-report-workshop

en.pdif(2009).

4. European Commission \& European Medicines Agency. European Commission-European Medicines Agency Conference on the Operation of the Clinical Trials

Directive (Directive 2001/20/EC) and Perspectives for the Future. EORTC (European Organisation for

Research and Treatment of Cancer) [online], http://

www.eortc.be/services/doc/EUCTD/EC-EMEA report CT 20071003.pdf (2007)

5. Heads of Medicines Agencies (HMA). Guidance document for a Voluntary Harmonisation Procedure

(VHP) for the assessment of multinational Clinical Trial Applications. Heads of Medicines Agencies (HMA)

[online], http://www.hma.eu/uploads/media/VHP

version_2 March 2010.pdf (2010).

6. Krafft, H. One voice for clinical trials in Europe

Regulatory Rapporteur 7, 21-24 (2010).

Acknowledgements

Merck Serono thank P. Delgado-Romero for her valuable scientific and editorial input during the course of the preparation of this publication.

Competing interests statement

The authors declare no competing financial interests. 